\title{
CLEC3B protects H9c2 cardiomyocytes from apoptosis caused by hypoxia via the PI3K/Akt pathway
}

\author{
Fenghua LV (i), Zhuo Wang (i), Yanli Huang (i), Aoyang Si 1 , and Yulei Chen (i) \\ Department of Cardiology, The First Affiliated Hospital of Xinxiang Medical University, Xinxiang, Henan, China
}

\begin{abstract}
Ischemic heart disease (IHD) is one of the leading causes of death worldwide. C-type lectin domain family 3 member B (CLEC3B) is a C-type lectin superfamily member and is reported to promote tissue remodeling. The serum levels of CLEC3B are downregulated in patients with cardiovascular disease. However, the molecular mechanisms of CLEC3B in IHD is not wellcharacterized. Therefore, we overexpressed CLEC3B and silenced CLEC3B in H9c2 rat cardiomyocytes for the first time. We then constructed a model of IHD in vitro through culturing H9c2 cardiomyocytes in serum-free medium under oxygen-deficit conditions. Then, Cell Counting Kit-8 (CCK-8), flow cytometry, qRT-PCR, and western blot assays were performed to investigate cell viability, apoptosis, and expression levels of CLEC3B, phosphatidylinositol 3-kinase (PI3K), phosphorylated PI3K (p-PI3K), protein kinase B (Akt), phosphorylated Akt (p-Akt), and cleaved-caspase 3. We observed that the mRNA expression of CLEC3B was decreased in hypoxic H9c2 cardiomyocytes $(P<0.05)$. Overexpression of CLEC3B increased cell viability $(P<0.01)$, inhibited cell apoptosis $(P<0.05)$, upregulated the levels of $p-P I 3 K / P I 3 K$ and $p-A k t / A k t(P<0.01$ or $P<0.05)$, and downregulated expression of cleaved-caspase $3(P<0.001)$ in hypoxic $\mathrm{H} 9 \mathrm{c} 2$ cardiomyocytes while silencing of CLEC3B caused the opposite results. Inhibition of the PI3K/Akt pathway reversed the protective effect of CLEC3B on hypoxic $\mathrm{H} 9 \mathrm{c} 2$ cardiomyocytes. Our study demonstrated that CLEC3B alleviated the injury of hypoxic H9c2 cardiomyocytes via the PI3K/ Akt pathway.
\end{abstract}

Key words: Ischemic heart disease; H9c2; CLEC3B; PI3K/Akt pathway; Cell apoptosis

\section{Introduction}

Ischemic heart disease (IHD) refers to a disease caused by a decrease in myocardial blood supply, which leads to damage and changes in myocardial function and structure. IHD is one of the leading causes of death worldwide and causes a serious threat to human health $(1,2)$. Although the diagnosis and treatment of IHD have been greatly developed, the disease remains a major life-threatening challenge (3-5). Thus, there is an urgent need to study the mechanisms of IHD and find its potential therapeutic targets.

It is well-known that the PI3K/Akt signaling pathway regulates numerous cellular activities, including cell proliferation, apoptosis, and metabolism (6,7). An increasing number of studies have shown that the alleviation of hypoxic injury or hypoxia/reoxygenation-induced injury of cells is accompanied by activation of the PI3K/Akt signaling pathway $(8,9)$.

C-type lectin domain family 3 member B (CLEC3B) is one of the C-type lectin superfamily members and encodes tetranectin protein $(10,11)$. It locates in plasma, extracellular matrix, and exosomes (12). Tetranectin is a plasminogen-binding protein that promotes the activation of plasminogen, and it has been considered to affect tissue remodeling (13). CLEC3B has been reported to regulate several diseases. Overexpression of CLEC3B can promote fracture healing (13), inhibit neuronal apoptosis in Parkinson's disease (14), and inhibit the proliferation of clear cell renal cell carcinoma (11). Furthermore, some studies revealed that serum levels of CLEC3B were downregulated in patients with cardiovascular disease $(15,16)$. However, the potential effects of CLEC3B on IHD progress are unclear.

In this study, we investigated the effects of CLEC3B on cell viability and apoptosis of $\mathrm{H} 9 \mathrm{c} 2$ cardiomyocytes under hypoxic conditions in vitro. We also explored the potential of CLEC3B signaling pathway in the hypoxia model. This study explored the regulation of CLEC3B in IHD through in vitro experiments and provided a new idea for the treatment of IHD. 


\section{Material and Methods}

\section{Materials}

PI3K/Akt pathway inhibitor LY294002 was purchased from Sigma Aldrich (USA). The Annexin V-APC/PI apoptosis kit was obtained from Abnova (China). RIPA lysis buffer was purchased from Beyotime (China) and the BCA protein assay kit was purchased from Thermo Scientific (USA). Cell Counting Kit-8 (CCK-8) was obtained from Dojindo (Japan). Antibodies against PI3K, p-PI3K, Akt, p-Akt, cleaved-caspase 3 , and $\beta$-actin were obtained from Abcam (UK). Efficient chemiluminescence (ECL) kit was obtained from Millipore (USA).

\section{Cell culture and treatment of hypoxia}

Rat H9c2 (2-1) cardiomyocytes were purchased from the Cell Bank of Type Culture Collection of Chinese Academy of Sciences (China). H9c2 cardiomyocytes were cultured in Dulbecco's modified eagle medium (DMEM) with $10 \%$ fetal bovine serum (FBS) (Hyclone, USA) and $1 \%$ penicillin-streptomycin (Sigma-Aldrich, USA). H9c2 cardiomyocytes were seeded into a $25-\mathrm{cm}^{2}$ cell culture bottle (Corning, USA) at a density of $1 \times 10^{4}$ cells $/ \mathrm{cm}^{2}$ and maintained at $37^{\circ} \mathrm{C}$ under a humidified atmosphere containing $5 \% \mathrm{CO}_{2}$. Then, $\mathrm{H} 9 \mathrm{c} 2$ cardiomyocytes were passaged at a $1: 2$ ratio when they reached $80 \%$ confluence. All experiments were performed using $\mathrm{H} 9 \mathrm{c} 2$ cardiomyocytes between 15 to 20 passage numbers.

For hypoxia, cells were grown to $80-90 \%$ confluence and then cultured under hypoxic conditions $\left(94 \% \mathrm{~N}_{2}, 5 \%\right.$ $\mathrm{CO}_{2}$, and $1 \% \mathrm{O}_{2}$ ) for $24 \mathrm{~h}$ at $37^{\circ} \mathrm{C}(17)$.

\section{Cell transfection}

PCR was used to amplify the complete coding fragment of CLEC3B. The fragment was connected into the pcDNA3.1 vector (Invitrogen, USA) to construct pcDNA 3.1-CLEC3B (pc-CLEC3B). The empty pcDNA3.1 was transfected as a negative control (pc-NC). Small interfering RNAs (siRNAs) against CLEC3B (si-CLEC3B) (F: 5'CAGUGUAGCUAUGUCUCCCAAGUCU-3', R: 5'-GACU UGGGAGACAUAGCUACACUG-3') and negative control siRNA (si-NC) (F: 5'-CAGCGAUGUAUCUCUAACCGGU UCU-3', R: 5'-AGAACCGGUUAGAGAUACAUCGCUG-3') were designed and synthesized by Invitrogen. Lipofectamine ${ }^{\circledR} 2000$ transfection reagent (Invitrogen) was used to perform cell transfection. H9c2 cardiomyocytes were seeded into 6-well plates, and transfection began when the cardiomyocytes reached $70-80 \%$ confluence.

For gene overexpression, pc-CLEC3B ( $2 \mu \mathrm{g} /$ well $)$ or pc-NC $(2 \mu \mathrm{g} /$ well $)$ and transfection reagent $(12 \mu \mathrm{L} /$ well $)$ was diluted in $150 \mu \mathrm{L}$ of serum-free Opti-MEM (Gibco, USA) for $5 \mathrm{~min}$ respectively. For gene silencing, si-CLEC3B (75 pmol/well) or si-NC (75 pmol/well) and transfection reagent $(7.5 \mu \mathrm{L} /$ well) were diluted in $100 \mu \mathrm{L}$ of serum- free Opti-MEM for $5 \mathrm{~min}$, respectively. Then, the diluted transfection reagent and plasmid/siRNA were mixed and incubated at $37^{\circ} \mathrm{C}$ for $30 \mathrm{~min}$. The transfection mixture was added to the cell culture medium and mixed. The cell culture medium was replaced with fresh DMEM containing $10 \%$ FBS after $6 \mathrm{~h}$ of transfection, and the culture was continued for $48 \mathrm{~h}$.

H9c2 cardiomyocytes were divided into 6 groups: control group (no hypoxia), hypoxia group (the cardiomyocytes underwent hypoxia as described above), pc-CLEC3B transfected cardiomyocytes cultured under hypoxia (pc-CLEC3B + hypoxia) group, pc-NC transfected cardiomyocytes cultured under hypoxia (pc-NC + hypoxia) group, si-CLEC3B transfected cardiomyocytes cultured under hypoxia (si-CLEC3B + hypoxia) group, and si-NC transfected cardiomyocytes cultured under hypoxia (si-NC + hypoxia) group. In order to further investigate the potential mechanisms of the effects of the pc-CLEC3B in H9c2 cardiomyocytes, an additional experiment was performed in which pc-CLEC3B was transferred into H9c2 cardiomyocytes with or without LY294002, an inhibitor of PI3K/ Akt, and cultured under hypoxia. Each experiment was performed in triplicate.

\section{Cell proliferation}

Cell proliferation was measured using the CCK-8 assay. H9c2 cardiomyocytes (6,000/well) were seeded into 96-well plates for $24 \mathrm{~h}$. Then, cells were incubated in 10\% CCK-8 solution for $1 \mathrm{~h}$, and absorbance values were measured at $450 \mathrm{~nm}$ using a microplate reader (Thermo Fisher Scientific, USA). Each experiment was performed in triplicate.

\section{Apoptosis assay}

Annexin V-APC/PI (Abnova, KA3807) staining and flow cytometry were performed to detect apoptosis of $\mathrm{H} 9 \mathrm{c} 2$ cardiomyocytes. H9c2 cardiomyocytes $\left(1 \times 10^{6}\right.$ cells/well) were seeded into 6-well plates for $24 \mathrm{~h}$ and then transfected and hypoxic-treated as described above. $\mathrm{H} 9 \mathrm{c} 2$ cardiomyocytes were digested using $1 \mathrm{~mL}$ of $0.25 \%$ trypsin (EDTA free) (Beyotime, C0205) for 2 min. Next, $\mathrm{H} 9 \mathrm{c} 2$ cardiomyocytes were washed with PBS buffer solution and centrifuged at $150 \mathrm{~g}$ for $5 \mathrm{~min}$ at room temperature. Then, $\mathrm{H} 9 \mathrm{c} 2$ cardiomyocytes were resuspended in $500 \mu \mathrm{L}$ of $1 \times$ binding buffer $\left(1 \times 10^{6}\right.$ cells $\left./ \mathrm{mL}\right)$ and added to appropriate tubes $\left(1 \times 10^{5}\right.$ cells /tube). Five microliters of Annexin V-APC and $5 \mu \mathrm{L}$ of PI solution were added to each tube for $15 \mathrm{~min}$ at room temperature in the dark. Subsequently, the cells were analyzed using a FACSCalibur $^{\mathrm{TM}}$ Flow Cytometer flow cytometry (BD Biosciences, USA) within $1 \mathrm{~h}$. The total percentage of apoptotic cells was defined as the sum of both early apoptosis (Annexin $\mathrm{V}$-APC positive, PI negative) and late apoptosis (Annexin $\mathrm{V}-\mathrm{APC} / \mathrm{PI}$ positive). Each experiment was performed in triplicate. 


\section{Quantitative real-time PCR assay}

TRIzol (Invitrogen) was used to isolate total RNA from $\mathrm{H} 9 \mathrm{c} 2$ cardiomyocytes. RevertAid First Strand cDNA Synthesis Kit (Thermo Scientific, USA) was used to perform the reverse transcription (RT) reaction and TB Green ${ }^{\mathbb{R}}$ Premix Ex Taq ${ }^{\mathrm{TM}}$ II (TaKaRa, Japan) was used to perform the qRT-PCR assay. The RT-qPCR results were analyzed as the fold change $\left(2^{-\Delta \Delta C t}\right)$ and normalized with the expression of $\beta$-actin. The sequences of primers were synthesized, as listed in Table 1 (Tsingke, China). Each experiment was performed in triplicate.

\section{Western blot analysis}

H9c2 cardiomyocytes were lysed with RIPA buffer for 30 min and quantified with a BCA protein assay kit. Equal amounts of protein samples were separated by $12 \%$ SDSPAGE gels and then transferred onto a polyvinylidene difluoride (PVDF) membrane (Millipore). After blocking with $5 \%$ skim milk for $1 \mathrm{~h}$ at room temperature, the membranes were incubated overnight at $4^{\circ} \mathrm{C}$ with primary

Table 1. Primer information.

\begin{tabular}{ll}
\hline Primer & \multicolumn{1}{c}{ Sequence $\left(5^{\prime}\right.$ to $\left.3^{\prime}\right)$} \\
\hline CLEC3B & F: ACGCCGCAGTCTGAGCTAGAGAATGA \\
& R: CGCCTTCCGAGCCATGTCGTTGAG \\
$\beta$-actin & F: GAAGATCAAGATCATTGCTCC \\
& R: TACTCCTGCTTGCTGATCCA \\
\hline
\end{tabular}

F: forward; R: reverse. antibodies cleaved-caspase 3 (ab2302, 1:1,000 dilution), PI3K (ab191606, 1:1,000 dilution), p-PI3K (ab182651, 1:1,000 dilution), Akt (ab81283, 1:5,000 dilution), p-Akt (ab81283, 1:1,000 dilution), CLEC3B (ab202134, 1:1,000 dilution), and $\beta$-actin (ab8226, 1:1,000 dilution). Goat antirabbit IgG H\&L secondary antibody (ab 205719, 1:2,000 dilution) was incubated with membranes at room temperature for $1 \mathrm{~h}$. Bands of protein were visualized using the ECL kit (WBULS0500). Relative protein expression was quantified using Image-ProPlus 6.0 software (Media Cybernetics, USA) and normalized with the expression of $\beta$-actin. Each experiment was performed in triplicate.

\section{Statistical analysis}

The quantitative data of this study are reported as means \pm SD. Statistical analysis was evaluated by SPSS 22.0 (IBM, USA) using Student's $t$-test or one-way analysis of variance (ANOVA). $\mathrm{P}<0.05$ was considered to indicate a statistically significant difference.

\section{Results}

\section{CLEC3B was downregulated in $\mathrm{H} 9 \mathrm{c} 2$ cardiomyocytes with hypoxia}

Cell viability of the hypoxia group was significantly decreased compared with the control group $(P<0.01)$ (Figure 1A). There was a significant increase in apoptosis (Figure 1B) after $24 \mathrm{~h}$ of hypoxia compared with the cells cultured normally $(P<0.01)$. The expression of CLEC3B was significantly decreased in the hypoxia group compared with the control group $(P<0.05)$ (Figure $1 \mathrm{C}$ and $\mathrm{D})$.
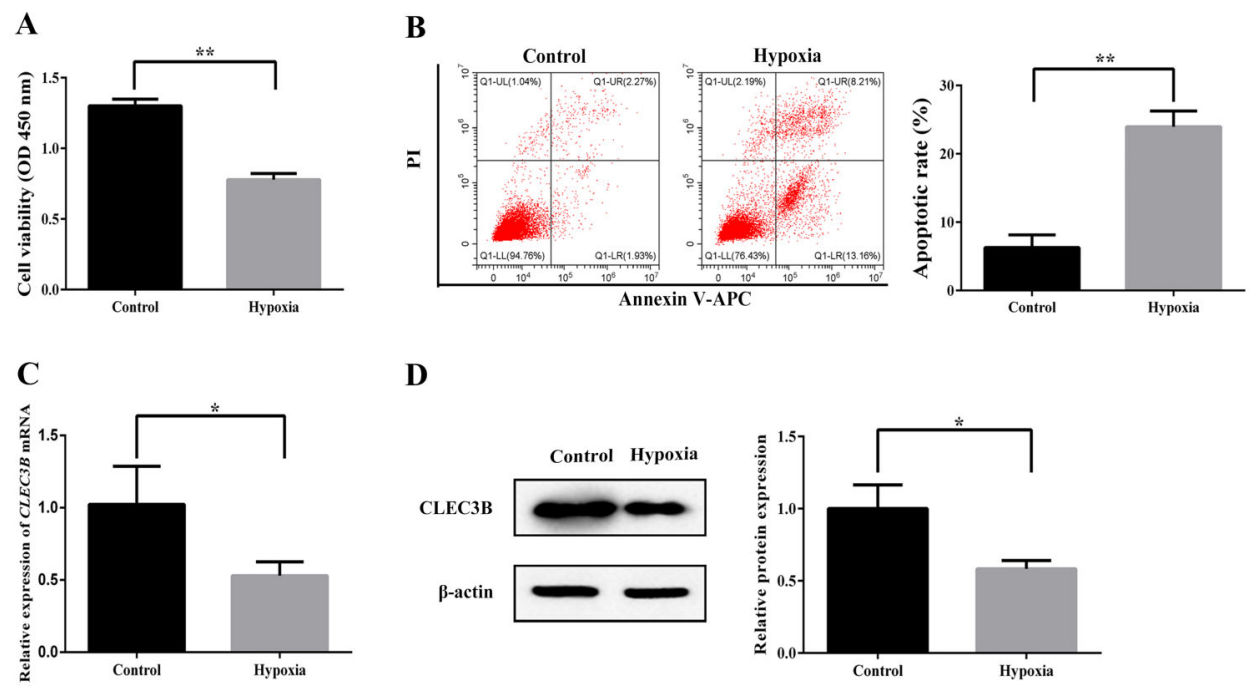

D
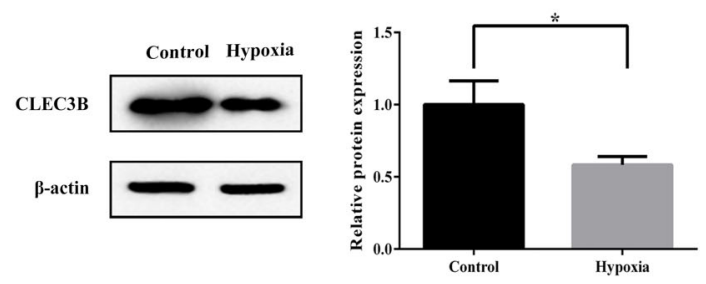

Figure 1. Effect of hypoxia on H9c2 cells. A, CCK8 assay was used to determine the effect of hypoxia on cell viability of H9c2 cells. B, Flow cytometry was used to evaluate the effect of hypoxia on apoptosis of H9c2 cells. C, QRT-PCR assay was performed to investigate the effect of hypoxia on CLEC3B mRNA expression in H9c2 cells. D, Western blot assay was used to detect the effect of hypoxia on the CLEC3B protein level in H9c2 cells. Data are reported as means \pm SD of 3 independent experiments. ${ }^{*} P<0.05$, ${ }^{* *} P<0.01$ (Student's $t$-test). 


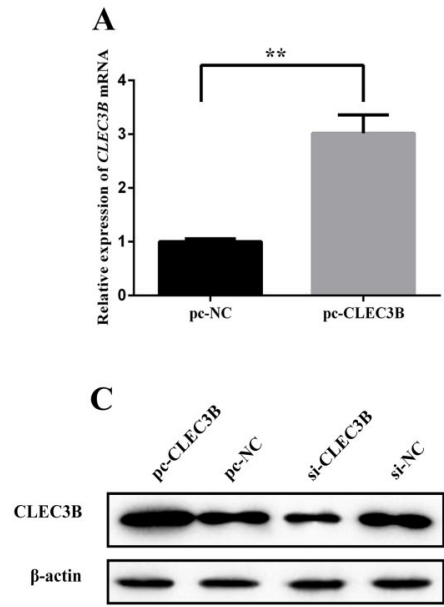

D

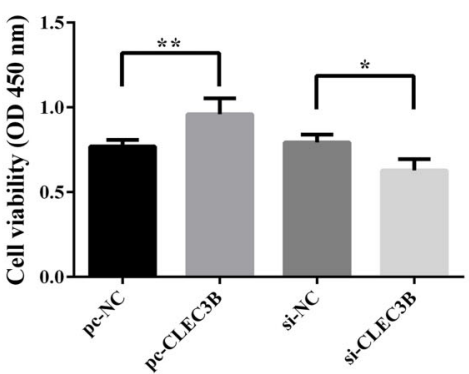

B
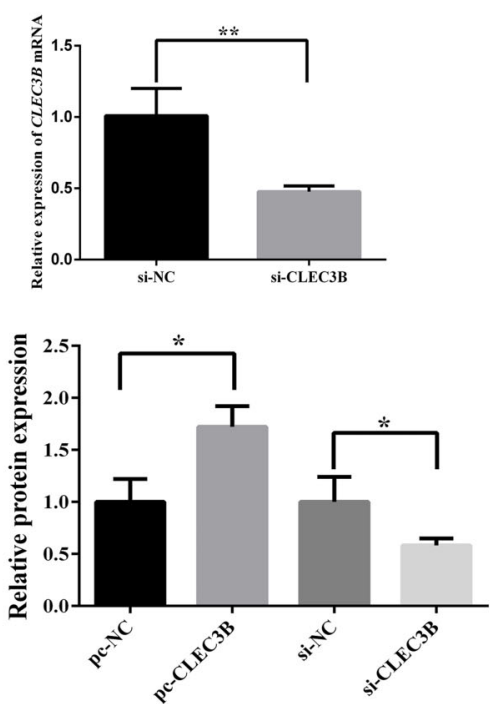

Figure 2. Effect of CLEC3B on cell viability of $\mathrm{H} 9 \mathrm{C} 2$ cells with oxygen deficit. $\mathbf{A}, \mathbf{B}$, and $\mathbf{C}$, Efficiencies of transfection of pc-CLEC3B and si-CLEC3B were verified by qRT-PCR and western blot assays. D, Cell viability of pc-NC (negative control), pc-CLEC3B, si-NC, or si-CLEC3B transfected H9c2 cells by CCK-8 assay. Data are reported as means \pm SD of 3 independent experiments. ${ }^{*} P<0.05$, ${ }^{* *} \mathrm{P}<0.01$ (Student's $t$-test or ANOVA).

These results indicated that the gene and protein expression levels of CLEC3B were decreased in H9c2 cardiomyocytes under hypoxia.

\section{Overexpression of CLEC3B promoted cell viability while loss of function of CLEC3B inhibited cell viabilityin hypoxic $\mathrm{H} 9 \mathrm{c} 2$ cardiomyocytes}

CLEC3B expression levels were significantly upregulated in the pc-CLEC3B group compared to the pc-NC group (Figure $2 \mathrm{~A}$ and $\mathrm{C}$ ). Meanwhile, the expression levels of CLEC3B were downregulated in the si-CLEC3B group compared with the si-NC group (Figure 2B and $\mathrm{C}$ ). Moreover, CCK8 assay was used to determine the effect of CLEC3B on cell viability of $\mathrm{H} 9 \mathrm{c} 2$ cardiomyocytes with oxygen deficit. As shown in Figure 2D, the cell viability of $\mathrm{H} 9 \mathrm{c} 2$ cardiomyocytes was increased in the pc-CLEC3B group compared with the pc-NC group, while the cell viability of $\mathrm{H} 9 \mathrm{c} 2$ cardiomyocytes was decreased in the si-CLEC3B group compared with the si-NC group. Our data demonstrated that transfection of pc-CLEC3B successfully increased cell viability of $\mathrm{H} 9 \mathrm{c} 2$ cardiomyocytes with hypoxia while transfection of si-CLEC3B successfully decreased cell viability of $\mathrm{H} 9 \mathrm{c} 2$ cardiomyocytes under hypoxia.

\section{Overexpression of CLEC3B inhibited apoptosis in hypoxic $\mathrm{H} 9 \mathrm{c} 2$ cardiomyocytes}

Apoptosis of $\mathrm{H} 9 \mathrm{c} 2$ cardiomyocytes was decreased in the pc-CLEC3B group compared with the pc-NC group $(P<0.01)$. Compared with the si-NC group, apoptosis of $\mathrm{H} 9 \mathrm{c} 2$ cardiomyocytes was increased in the si-CLEC3B group $(P<0.01)$ (Figure 3 ).

\section{CLEC3B activated PI3K/Akt signaling pathway and decreased cleaved-caspase 3 protein expression in hypoxic $\mathrm{H} 9 \mathrm{c} 2$ cardiomyocytes}

Among the $\mathrm{H} 9 \mathrm{c} 2$ cardiomyocytes from the hypoxia group, the levels of $\mathrm{p}-\mathrm{PI} 3 \mathrm{~K} / \mathrm{PI} \mathrm{K}$ and $\mathrm{p}-\mathrm{Akt} / \mathrm{Akt}$ were markedly decreased compared with the control group $(P<0.01)$, 

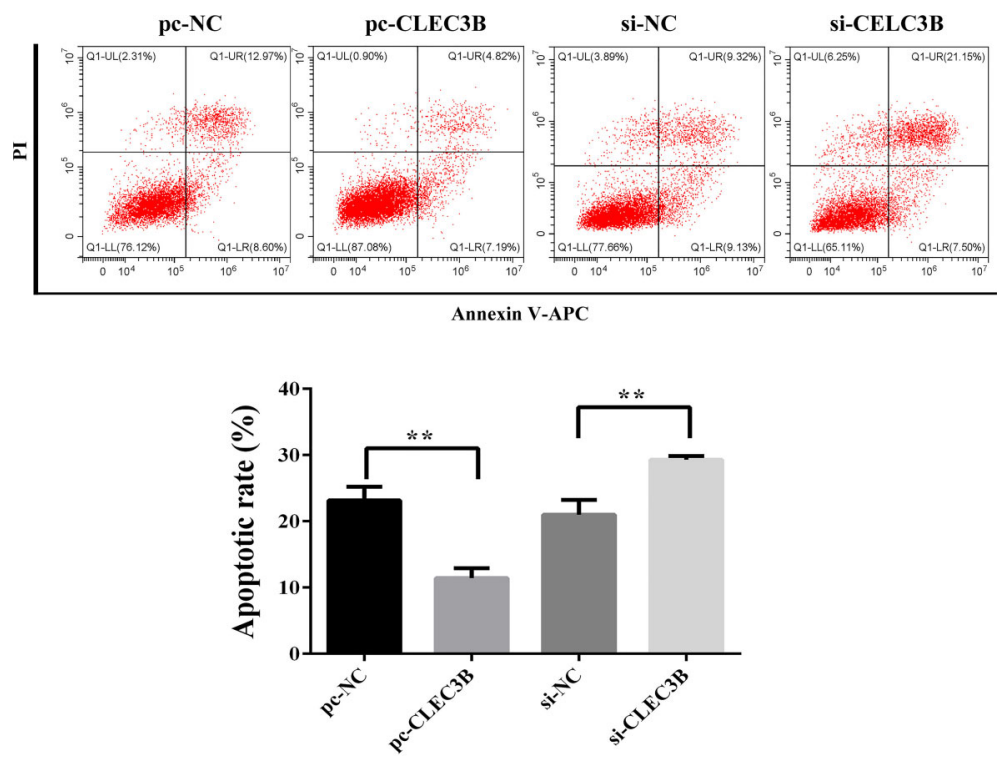

Figure 3. Effect of CLEC3B on apoptosis of H9c2 cardiomyocytes. Annexin V-APC/PI staining and flow cytometry were used to evaluate the effect of CLEC3B on apoptosis of H9c2 cardiomyocytes with hypoxia. Data are reported as means \pm SD of 3 independent experiments. ${ }^{* *} \mathrm{P}<0.01$ (ANOVA).
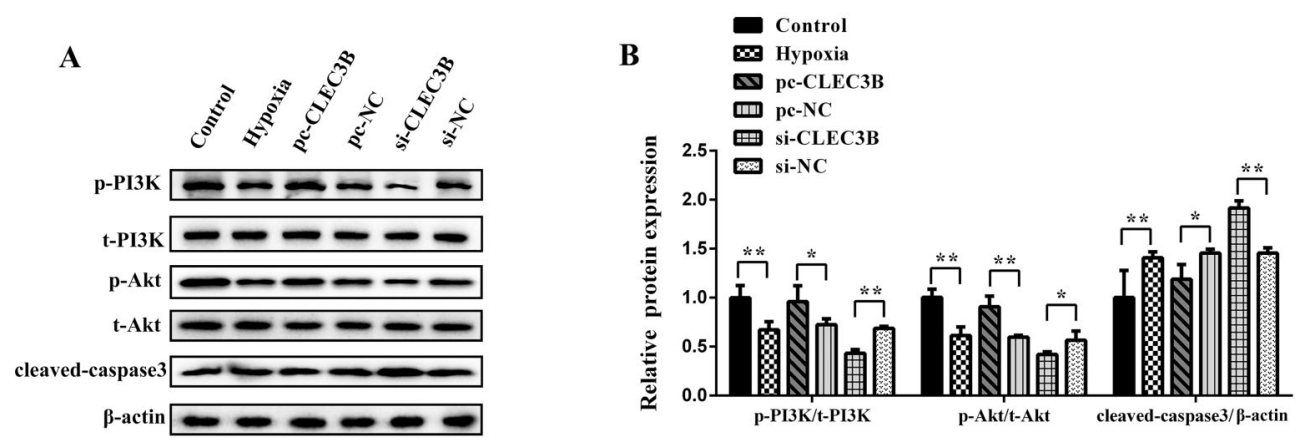

Figure 4. Effect of CLEC3B on the PI3K/Akt signaling pathway. A, Cells were transfected with pc-NC (negative control), pc-CLEC3B, siNC, or si-CLEC3B and cultured in serum-free medium with hypoxia. Protein expression was evaluated by western blot analysis. B, Activation of the PI3K/Akt pathway was shown as the relative intensity of phosphorylated proteins/total proteins, and the relative intensity of cleaved-caspase 3 was normalized by $\beta$-actin. Data are reported as means $\pm S D$ of 3 independent experiments. ${ }^{*} P<0.05$, ${ }^{* *} \mathrm{P}<0.01$ (ANOVA).

while the expression of cleaved-caspase 3 was increased compared with the control group $(P<0.01)$. Levels of $\mathrm{p}-\mathrm{PI} 3 \mathrm{~K} / \mathrm{PI} \mathrm{KK}$ and $\mathrm{p}-\mathrm{Akt} / \mathrm{Akt}$ were significantly increased $(P<0.05$ or $P<0.01)$ while the expression level of cleavedcaspase 3 was decreased $(P<0.05)$ by CLEC3B overexpression compared with the pc-NC group. At the same time, compared with the si-NC group, knockdown of CLEC3B decreased the levels of p-PI3K/PI3K and p-Akt/ Akt $(P<0.01$ or $P<0.05)$ and upregulated the expression of cleaved-caspase $3(P<0.01)$ (Figure $4 A$ and $B$ ). These data indicated that the effects of CLEC3B on hypoxia of $\mathrm{H} 9 \mathrm{c} 2$ cardiomyocytes could be connected with the activation of the PI3K/Akt signaling pathway and the inhibition of cleaved-caspase 3 , an apoptosis-related protein.

\section{Inhibition of PI3K/Akt signaling pathway reversed the anti-apoptotic influence of CLEC3B}

The level of p-Akt/Akt was decreased while the expression of cleaved-caspase 3 was increased upon treatment with LY294002 compared with the pc-CLEC3B group $(P<0.05)$ (Figure $5 A$ and $B)$. The apoptosis rate was significantly increased in the pc-CLEC3B + LY294 002 group compared with the pc-CLEC3B group $(\mathrm{P}<0.01)$ (Figure 5C). 

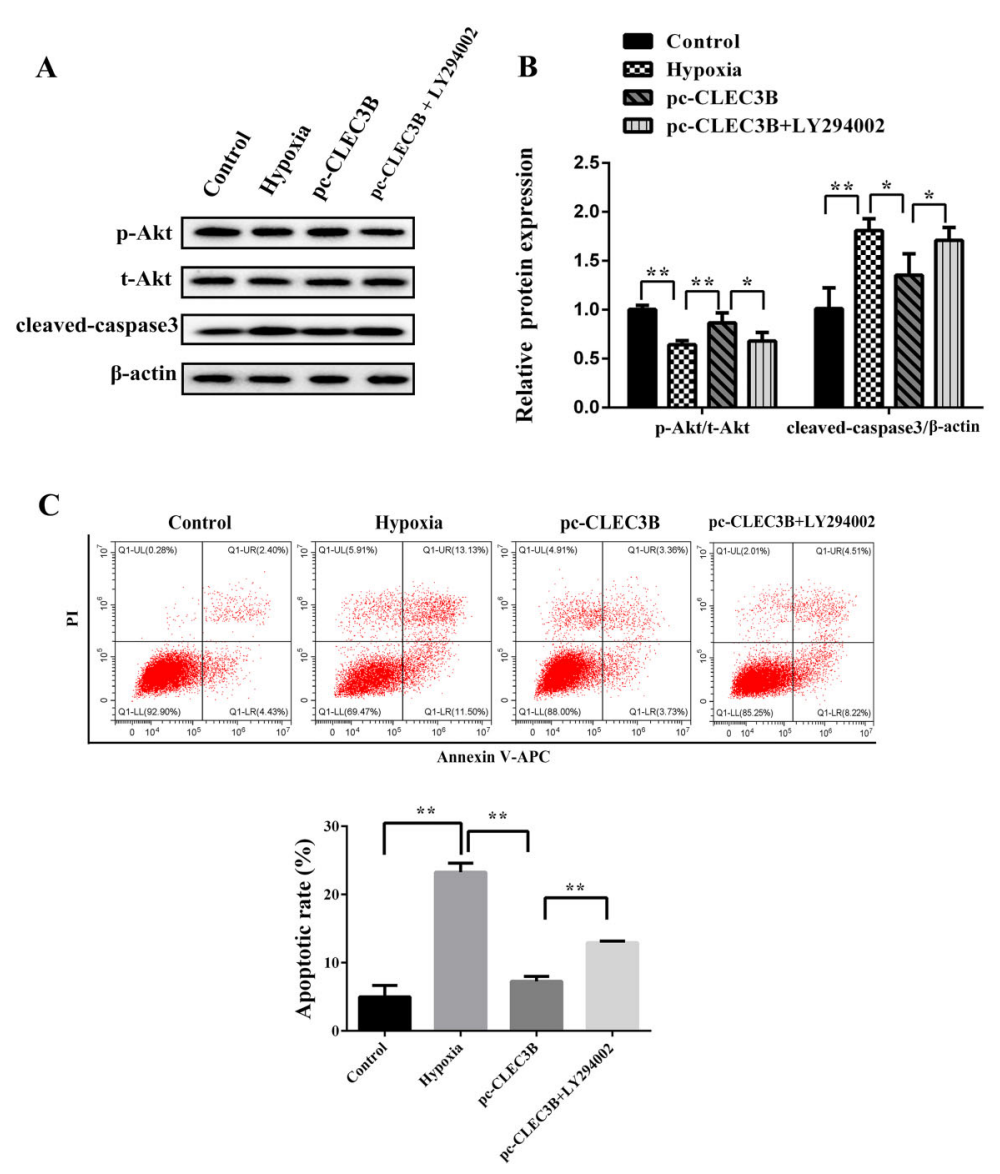

Figure 5. Effect of the inhibition of the PI3K/Akt signaling pathway on apoptosis of $\mathrm{H} 9 \mathrm{C} 2$ cells. $\mathbf{A}$ and $\mathbf{B}$, Western blot was performed to evaluate the effect of LY294002 on the PI3K/Akt signaling pathway. C, Annexin V-APC/PI staining and flow cytometry were used to evaluate the effect of the inhibition of the PI3K/Akt signaling pathway on apoptosis of H9c2 cells. Data are reported as means \pm SD of 3 independent experiments. ${ }^{*} \mathrm{P}<0.05,{ }^{* *} \mathrm{P}<0.01$ (ANOVA).

\section{Discussion}

IHD is still one of the most dangerous diseases causing human death, and finding a therapeutic target for IHD is crucial $(18,19)$. To investigate the molecular mechanisms of CLEC3B in IHD, H9c2 cardiomyocytes were used to simulate myocardial ischemia (20). Many factors affect the development of IHD $(20,21)$. CLEC3B encodes tetranectin, which binds to plasminogen in a lysine-dependent manner and promotes the activation of plasminogen to regulate proteolytic processes $(11,22)$. Here, we focused on CLEC3B and explored the role of CLEC3B in H9c2 cardiomyocytes under hypoxic conditions. Our results indicated that CLEC3B alleviated the injury of hypoxic H9c2 cardiomyocytes via the PI3K/Akt pathway.

Several studies reported that CLEC3B promotes myogenesis and inhibits cancer cell proliferation $(11,23)$. In addition, the serum level of CLEC3B was downregulated in patients with coronary artery disease (16).
Meanwhile, the expression level of CLEC3B was increased in hypoxic myocardial cells treated by miR-19a/19b mimics, two protective miRNAs of myocardial infarction (24). In this study, the protein and mRNA expression of CLEC3B were decreased in hypoxic H9c2 cardiomyocytes. Our data indicated, for the first time, that overexpression of CLEC3B increased cell viability and decreased apoptosis in hypoxic H9c2 cardiomyocytes.

It is well accepted that the PI3K/Akt pathway regulates cell proliferation, apoptosis, and metabolism (25). Liu et al. (26) discovered that isoflurane alleviates oxygen-glucose deprivation-induced $\mathrm{H} 9 \mathrm{c} 2$ cell injury via activation of the PI3K/Akt pathway. Zhang et al. (19) found that emodin protects H9c2 cardiomyocytes from hypoxic injury by upregulating the expression of miR-138, which targeted mixed lineage kinase 3 (MLK3) and increased p-Akt expression. Therefore, the PI3K/Akt pathway might be a potential target for the treatment of myocardial ischemia. Moreover, cleaved-caspase 3 is an apoptosis-activating 
protein, which is regulated by the PI3K/Akt pathway and serves as a marker for the apoptosis-promoting effect $(27,28)$. Therefore, we conjectured that the effect of CLEC3B on the proliferation and apoptosis of hypoxic $\mathrm{H} 9 \mathrm{c} 2$ cardiomyocytes might be connected with the PI3K/ Akt pathway and the expression of cleaved-caspase 3 . Our data further demonstrated that the PI3K/Akt pathway was inhibited, and cleaved-caspase 3 expression was increased by oxygen deprivation in $\mathrm{H} 9 \mathrm{c} 2$ cardiomyocytes. Overexpression of CLEC3B activated the PI3K/Akt pathway and reduced the expression of cleaved-caspase 3 while the silencing of CLEC3B caused the opposite results. Inhibition of the PI3K/Akt pathway reversed the protective effect of CLEC3B on hypoxic H9c2 cardiomyocytes.

\section{References}

1. Finegold JA, Asaria P, Francis DP. Mortality from ischaemic heart disease by country, region, and age: statistics from World Health Organisation and United Nations. Int J Cardiol 2013; 168: 934-945, doi: 10.1016/j.ijcard.2012.10.046.

2. Han XD, Zhou ZW, Yang W, Ye HC, Xu YZ, Huang YF, et al. A computational and functional study elicits the ameliorating effect of the Chinese herbal formula Huo Luo Xiao Ling Dan on experimental ischemia-induced myocardial injury in rats via inhibition of apoptosis. Drug Des Devel Ther 2015; 9: 1063-1102, doi: 10.2147/DDDT.S76336.

3. Gerczuk PZ, Kloner RA. An update on cardioprotection: a review of the latest adjunctive therapies to limit myocardial infarction size in clinical trials. J Am Coll Cardiol 2012; 59: 969-978, doi: 10.1016/j.jacc.2011.07.054.

4. Hausenloy DJ, Botker HE, Engstrom T, Erlinge D, Heusch $\mathrm{G}$, Ibanez B, et al. Targeting reperfusion injury in patients with ST-segment elevation myocardial infarction: trials and tribulations. Eur Heart J 2017; 38: 935-941, doi: 10.1093/ eurheartj/ehw145.

5. Cung TT, Morel O, Cayla G, Rioufol G, Garcia-Dorado D, Angoulvant $\mathrm{D}$, et al. Cyclosporine before $\mathrm{PCl}$ in patients with acute myocardial infarction. N Engl J Med 2015; 373: 10211031, doi: 10.1056/NEJMoa1505489.

6. Li L, Qu Y, Mao M, Xiong Y, Mu D. The involvement of phosphoinositid 3-kinase/Akt pathway in the activation of hypoxia-inducible factor-1alpha in the developing rat brain after hypoxia-ischemia. Brain Res 2008; 1197: 152-158, doi: 10.1016/j.brainres.2007.12.059.

7. Zhang Z, Yao L, Yang J, Wang Z, Du G. PI3K/Akt and HIF-1 signaling pathway in hypoxia-ischemia (Review). Mol Med Rep 2018; 18: 3547-3554, doi: 10.3892/mmr.2018.9375.

8. Zhang C, Pan S, Aisha A, Abudoukelimu M, Tang L, Ling Y. Recombinant human brain natriuretic peptide regulates $\mathrm{PI} 3 \mathrm{~K} / \mathrm{AKT} / \mathrm{mTOR}$ pathway through IncRNA EGOT to attenuate hypoxia-induced injury in $\mathrm{H} 9 \mathrm{c} 2$ cardiomyocytes. Biochem Biophys Res Commun 2018; 503: 1186-1193, doi: 10.1016/j.bbrc.2018.07.023.

9. Xiao JM, Wang JJ, Sun LL. Effect of miR-134 against myocardial hypoxia/reoxygenation injury by directly targeting NOS3 and regulating PI3K/Akt pathway. Acta Cir Bras 2019; 34: e201900802, doi: 10.1590/s0102-86502019008 0000002.
In conclusion, this study indicated that CLEC3B was decreased in hypoxic $\mathrm{H} 9 \mathrm{c} 2$ cardiomyocytes and it protected hypoxic $\mathrm{H} 9 \mathrm{c} 2$ cardiomyocytes from apoptosis via the PI3K/Akt pathway. We provided the first connection between CLEC3B and the PI3K/Akt pathway. These results help to clarify potential treatment targets for IHD. Further animal and clinical studies should be performed in the future to validate this mechanism.

\section{Acknowledgments}

This work was supported by a grant from the Realistic Exploration Fund of Heart Center, First Affiliated Hospital of Xinxiang Medical University (No. XZZX2017002).

10. Tanisawa K, Arai $\mathrm{Y}$, Hirose N, Shimokata H, Yamada $\mathrm{Y}$, Kawai $\mathrm{H}$, et al. Exome-wide association study identifies CLEC3B missense variant p.S106G as being associated with extreme longevity in east Asian populations. J Gerontol A Biol Sci Med Sci 2017; 72: 309-318, doi: 10.1093/gerona/ glw074.

11. Liu J, Liu Z, Liu Q, Li L, Fan X, Wen T, et al. CLEC3B is downregulated and inhibits proliferation in clear cell renal cell carcinoma. Oncology Rep 2018; 40: 2023-2035, doi: 10.3892/or.2018.6590.

12. Dai W, Wang Y, Yang T, Wang J, Wu W, Gu J. Downregulation of exosomal CLEC3B in hepatocellular carcinoma promotes metastasis and angiogenesis via AMPK and VEGF signals. Cell Commun Signa 2019; 17: 113, doi: 10.1186/s12964-019-0423-6.

13. Iba K, Abe Y, Chikenji T, Kanaya K, Chiba H, Sasaki K, et al. Delayed fracture healing in tetranectin-deficient mice. J Bone Miner Metab 2013; 31: 399-408, doi: 10.1007/ s00774-013-0436-y.

14. Chen Z, Wang E, Hu R, Sun $Y$, Zhang L, Jiang J, et al. Tetranectin gene deletion induces Parkinson's disease by enhancing neuronal apoptosis. Biochem Biophys Res Commun 2015; 468: 400-407, doi: 10.1016/j.bbrc.2015.10.118.

15. Yin X, Subramanian S, Hwang SJ, O'Donnell CJ, Fox CS, Courchesne $P$, et al. Protein biomarkers of new-onset cardiovascular disease: prospective study from the systems approach to biomarker research in cardiovascular disease initiative. Arterioscler Thromb Vasc Biol 2014; 34: 939-945, doi: 10.1161/ATVBAHA.113.302918.

16. Chen $Y$, Han $H$, Yan $X$, Ding $F$, Su $X$, Wang $H$, et al. Tetranectin as a potential biomarker for stable coronary artery disease. Sci Rep 2015; 5: 17632, doi: 10.1038/srep17632.

17. Zhang Z, Li H, Liu M, He J, Zhang X, Chen Y. Skullcapflavone I protects cardiomyocytes from hypoxia-caused injury through up-regulation of lincRNA-ROR. Int $\mathrm{J}$ Immunopathol Pharmacol 2019; 33: 2058738419857537, doi: 10.1177/2058738419857537.

18. Paine NJ, Bacon SL, Pelletier R, Arsenault A, Diodati JG, Lavoie KL. Do women with anxiety or depression have higher rates of myocardial ischemia during exercise testing than men? Circ Cardiovasc Qual Outcomes 2016; 9: S53S61, doi: 10.1161/CIRCOUTCOMES.115.002491. 
19. Zhang X, Qin Q, Dai H, Cai S, Zhou C, Guan J. Emodin protects $\mathrm{H} 9 \mathrm{c} 2$ cells from hypoxia-induced injury by upregulating miR-138 expression. Braz J Med Biol Res 2019; 52: e7994, doi: 10.1590/1414-431x20187994.

20. Long TY, Jing R, Kuang F, Huang L, Qian ZX, Yang TL. CIRBP protects $\mathrm{H} 9 \mathrm{C} 2$ cells against myocardial ischemia through inhibition of NF-kB pathway. Braz J Med Biol Res 2017; 50: e5861, doi: 10.1590/1414-431x20175861.

21. Buch $K$, Peters $T$, Nawroth $T$, Sanger $M$, Schmidberger $H$, Langguth P. Determination of cell survival after irradiation via clonogenic assay versus multiple MTT Assay--a comparative study. Radiat Oncol 2012; 7: 1, doi: 10.1186/1748-717X-7-1.

22. Clemmensen I, Petersen LC, Kluft C. Purification and characterization of a novel, oligomeric, plasminogen kringle 4 binding protein from human plasma: tetranectin. Eur $\mathrm{J}$ Biochem 1986; 156: 327-333, doi: 10.1111/j.1432-1033. 1986.tb09586.x.

23. Wewer UM, Iba K, Durkin ME, Nielsen FC, Loechel F, Gilpin BJ, et al. Tetranectin is a novel marker for myogenesis during embryonic development, muscle regeneration, and muscle cell differentiation in vitro. Dev Biol 1998; 200: 247259, doi: 10.1006/dbio.1998.8962.
24. Gao F, Kataoka M, Liu N, Liang T, Huang ZP, Gu F, et al. Therapeutic role of miR-19a/19b in cardiac regeneration and protection from myocardial infarction. Nat Commun 2019; 10: 1802, doi: 10.1038/s41467-019-09530-1.

25. Xie Y, Shi X, Sheng K, Han G, Li W, Zhao Q, et al. PI3K/Akt signaling transduction pathway, erythropoiesis and glycolysis in hypoxia (Review). Mol Med Rep 2019; 19: 783-791, doi: 10.3892/mmr.2018.9713.

26. Liu J, Yang S, Zhang X, Liu G, Yue X. Isoflurane reduces oxygen-glucose deprivation-induced oxidative, inflammatory, and apoptotic responses in $\mathrm{H} 9 \mathrm{c} 2$ cardiomyocytes. Am J Transl Res 2016; 8: 2597-2608.

27. $\mathrm{Xu} \mathrm{L}$, Jiang $\mathrm{X}$, Wei $\mathrm{F}, \mathrm{Zhu} \mathrm{H}$. Leonurine protects cardiac function following acute myocardial infarction through antiapoptosis by the PI3K/AKT/GSK3beta signaling pathway. Mol Med Rep 2018; 18: 1582-1590, doi: 10.3892/mmr.2018. 9084.

28. Zhang X, Wang L, Peng L, Tian X, Qiu X, Cao H, et al. Dihydromyricetin protects HUVECs of oxidative damage induced by sodium nitroprusside through activating PI3K/ Akt/FoxO3a signalling pathway. J Cell Mol Med 2019; 23: 4829-4838, doi: $10.1111 / \mathrm{jcmm} .14406$. 